\title{
Problemas com o sono e fatores associados na população brasileira: Pesquisa Nacional de Saúde, 2013
}

\author{
Sleep-related problems and associated factors among the Brazilian \\ population: National Health Survey, 2013
}

Yanka Zanolo Gajardo (https://orcid.org/0000-0002-8104-4405) ${ }^{1}$

Juliana Nunes Ramos (https://orcid.org/0000-0003-1069-8353) ${ }^{1}$

Ana Paula Muraro (https://orcid.org/0000-0001-6237-1673) ${ }^{2}$

Naiara Ferraz Moreira (https://orcid.org/0000-0002-5847-2831) ${ }^{3}$

Marcia Gonçalves Ferreira (https://orcid.org/0000-0001-8362-0819) ${ }^{1}$

Paulo Rogério Melo Rodrigues (https://orcid.org/0000-0002-4213-0685) ${ }^{1}$
${ }^{1}$ Faculdade de Nutrição, Universidade Federal de Mato Grosso (UFMT). Av. Fernando Corrêa da Costa 2367, Bairro Boa Esperança. 78060-900 Cuiabá MT Brasil.prmr84@gmail.com ${ }^{2}$ Instituto de Saúde Coletiva, UFMT. Cuiabá MT Brasil.

${ }^{3}$ Faculdade de Ciências da Saúde, Universidade Federal da Grande Dourados. Dourados MS Brasil.

\begin{abstract}
The scope of this study was to estimate the frequency of sleep-related problems and associated factors. It was a study based on data from the National Health Survey conducted in Brazil in 2013. Sleep-related problems were assessed with the question "How often have you had sleep problems in the last two weeks, such as difficulty falling asleep, waking up frequently during the night or sleeping more than usual?" The answers were grouped into: none of the days, less than half of the days and more than half of the days. Of the individuals evaluated, $71.1 \%$ reported having no problems with sleep on any day, $14.0 \%$ on at least half of the days and $14.9 \%$ on more than half of the days. The highest frequencies of sleep-related problems were reported by women and more advanced age. For men and women, smoking, alcohol abuse, physical inactivity, sedentary behavior, and high blood pressure were significantly associated with the frequency of sleep problems on more than half of the days, and also excess weight for women. Thus, the frequency of sleep-related problems in the Brazilian population was high. Women, older individuals with lifestyle-related risk behaviors, high blood pressure, and overweight were the highest risk subgroups.
\end{abstract}

Key words Sleep-related problems, Health conditions, Risk behaviors
Resumo O objetivo deste estudo foi estimar a frequência de problemas com o sono e os fatores associados, analisando dados da Pesquisa Nacional de Saúde, realizado em 2013 no Brasil. Os problemas com o sono foram avaliados com a pergunta "Nas duas últimas semanas, com que frequência o(a) $\operatorname{sr}(a)$ teve problemas no sono, como dificuldade para adormecer, acordar frequentemente à noite ou dormir mais do que de costume?", as respostas foram agrupadas em: nenhum dia, menos da metade dos dias e mais da metade dos dias. Dos indivíduos avaliados, $71,1 \%$ relataram não ter problemas com o sono em nenhum dia, $14,0 \% \mathrm{em}$ pelo menos metade dos dias e $14,9 \%$ em mais da metade dos dias. As maiores frequências de problemas com o sono foram relatadas pelas mulheres e com o aumento da idade. Para homens e mulheres, tabagismo, consumo de bebidas alcoólicas, inatividade fisica, comportamento sedentário $e$ hipertensão arterial apresentaram associação significativa com a frequência de problemas com o sono em mais da metade dos dias, e o excesso de peso para as mulheres. Assim, foi elevada a frequência de problemas com o sono na população brasileira. Mulheres, individuos mais velhos, com comportamentos de risco relacionados ao estilo de vida, hipertensão arterial e excesso de peso corporal foram os subgrupos em maior risco.

Palavras-chave Problemas com o sono, Condições de saúde, Comportamentos de risco 


\section{Introdução}

O sono é um estado vital e complexo, caracterizado por processos ativos e organizados, sendo essencial para a sobrevivência e considerado uma necessidade biológica crucial para todos os indivíduos por ser um restaurador físico e mental ${ }^{1}$. Por outro lado, a privação do sono pode causar alterações comportamentais e fisiológicas que colocam a vida em risco $^{2}$. Nesse sentido, as perturbações do sono (despertares no meio da noite, pesadelos, entre outros) ${ }^{3}$ podem acarretar alterações significativas no funcionamento físico, ocupacional, cognitivo e social do indivíduo, comprometendo substancialmente a qualidade de vida ${ }^{4}$, além interferir de modo negativo na atuação profissional do indivíduo, aumentando o risco de acidentes de trabalho e pessoais ${ }^{5,6}$.

A qualidade e o tempo de sono podem ser afetados por diversos fatores culturais, sociais, psicológicos, comportamentais, fisiopatológicos e ambientais ${ }^{7,8}$. Adicionalmente, comportamentos de risco relacionados ao estilo de vida, tais como tabagismo, inatividade física, consumo de bebidas alcoólicas e comportamentos sedentários, também podem apresentar efeito negativo sobre o sono ${ }^{9-11}$.

Alterações no sono têm sido consideradas tanto sinal preditivo de diversas doenças, quanto sintoma. Nesse sentido, tais alterações estão associadas a distúrbios metabólicos, representado importante fator de risco para o desenvolvimento de obesidade ${ }^{12,13}$, doenças metabólicas e cardiovasculares ${ }^{2,14,15}$ e resistência à insulina e diabetes $^{2,14,16}$.

No Brasil, em 2008, uma pesquisa nacional com amostra de 150 cidades indicou que 63\% dos indivíduos avaliados relataram pelo menos uma queixa relacionada ao sono ${ }^{17}$. Em 2012, em estudo realizado com amostra representativa da população (indivíduos com $>16$ anos de 132 cidades) foi verificado que $76 \%$ dos indivíduos apresentavam pelo menos um problema relacionado ao sono ${ }^{18}$.

Nesse contexto, considerando que o sono exerce grande influência na saúde e qualidade de vida das pessoas, o objetivo do presente estudo foi estimar a frequência de problemas com o sono e os fatores associados na população brasileira, utilizando dados da última Pesquisa Nacional de Saúde (PNS 2013).

\section{Métodos}

No presente trabalho foram analisados dados obtidos na Pesquisa Nacional de Saúde (PNS), inquérito domiciliar de base populacional conduzido em 2013 pelo Instituto Brasileiro de Geografia e Estatística (IBGE) em parceria com o Ministério da Saúde. A PNS é parte integrante do Sistema Integrado de Pesquisas Domiciliares do IBGE e utiliza a amostra mestra desse sistema, de maior cobertura geográfica e ganho de precisão nas estimativas. Com desenho próprio, a PNS foi elaborada especificamente para coletar informações de saúde ${ }^{19,20}$.

O plano amostral empregado foi de amostragem probabilística em três estágios. No primeiro estágio, houve estratificação das unidades primárias de amostragem (UPA), constituídas pelos setores ou conjunto de setores censitários. Os domicílios formaram as unidades de segundo estágio, e os moradores com 18 e mais anos de idade corresponderam às unidades de terceiro estágio. A seleção da subamostra de UPA foi feita por amostragem aleatória simples. A amostra foi estimada em 81.167 domicílios e foram coletadas informações em 64.348 deles, onde foram entrevistados 60.202 adultos. Considerando-se os domicílios fechados, a proporção de perdas foi de $20,8 \%$, e a proporção de não resposta, de $8,1 \%{ }^{19}$. Foram definidos pesos amostrais para as UPA, os domicílios e todos seus moradores; e um peso para o morador selecionado. Dessa forma, as estimativas fornecidas pela PNS são ponderadas considerando (i) o peso da UPA em relação à probabilidade de fazer parte da pesquisa, (ii) o peso do domicílio, com ajustes para correção de não resposta e calibração pelos totais populacionais, e (iii) o peso do morador selecionado, considerando a probabilidade de seleção do morador, ajustes de não resposta por sexo e calibração pelos totais populacionais por sexo e faixas etárias estimados com o peso de todos os moradores $^{19,20}$.

Para a coleta de dados os entrevistados responderam ao questionário com registro por computadores de mão (Personal Digital Assistan$c e$ - PDA). Após o contato com a pessoa responsável ou com algum dos moradores do domicílio selecionado, o entrevistador descreveu para o morador os objetivos e procedimentos do estudo, e a importância de sua participação na pesquisa, sendo elaborada uma lista de todos os moradores adultos do domicílio. Foram identificados o informante, que respondeu ao questionário domiciliar, e todos os moradores do domicílio, além 
do morador adulto encarregado de responder à entrevista individual, selecionado por programa de seleção aleatória no PDA. As entrevistas foram agendadas nas datas e horários mais convenientes para os informantes, prevendo-se duas ou mais visitas a cada domicílio ${ }^{19}$. Após a aplicação do questionário, foram aferidos peso, altura, circunferência da cintura e pressão arterial, sendo utilizados, respectivamente, balança eletrônica portátil, estadiômetro portátil, fita antropométrica e aparelho de pressão digital ${ }^{21}$.

Os problemas com o sono foram avaliados com a pergunta: "Nas duas últimas semanas, com que frequência o(a) $\operatorname{sr}(\mathrm{a})$ teve problemas no sono, como dificuldade para adormecer, acordar frequentemente à noite ou dormir mais do que de costume?" com as seguintes opções de resposta: "Nenhum dia", "Menos da metade dos dias", "Mais da metade dos dias" ou "Quase todos os dias". Para as análises estatísticas, as categorias "Mais da metade dos dias" e "Quase todos os dias" foram agrupadas em "Mais da metade dos dias".

No presente estudo foram avaliadas como variáveis independentes o sexo (masculino, feminino), a faixa etária ( 18 a 24, 25 a 39, 40 a 59 e 60 e mais anos) e os comportamentos de risco relacionados ao estilo de vida, considerando:

a) Tabagismo: foi considerado fumante, o indivíduo fumante atual, que relatou fumar, independentemente da quantidade e regularidade através da pergunta: "Atualmente, o(a) $\operatorname{sr}(\mathrm{a})$ fuma algum produto do tabaco?".

b) Consumo abusivo de bebida alcoólica: o consumo abusivo de álcool foi definido pela afirmativa à pergunta: "Nos últimos 30 dias, o sr. chegou a consumir 5 ou mais doses de bebida alcoólica em uma única ocasião?”. Para mulheres, é considerado consumo abusivo de álcool 4 ou mais doses.

c) Inatividade física: foram considerados insuficientemente ativos os indivíduos que não praticaram atividade física ou praticaram por menos do que 150 minutos por semana considerando os três domínios: lazer, trabalho e deslocamento para o trabalho.

d) Comportamento sedentário: foi avaliado mediante a pergunta: "Em média, quantas horas por dia o(a) $\operatorname{sr}($ a) costuma ficar assistindo televisão?". Foi considerado sedentário o indivíduo que passou três ou mais horas vendo televisão.

Adicionalmente, foram avaliadas como variáveis independentes as condições de saúde apresentadas pelos indivíduos, considerando hipertensão arterial e excesso de peso corporal.
As medidas de peso e estatura foram aferidas seguindo procedimentos padronizados, sendo o peso registrado em $\mathrm{kg}$ e medido por meio de balança portátil e digital com capacidade para 150 $\mathrm{kg}$, e para aferição da altura utilizou-se estadiômetro portátil. Essas medidas foram utilizadas para calcular o Índice de Massa Corporal (IMC= peso/estatura ${ }^{2}$ ), sendo a condição de peso classificada de acordo com a faixa etária: adolescentes (indivíduos de 18 e 19 anos de idade) ${ }^{22}$, adultos (de 20 a 59 anos de idade ${ }^{23}$ e idosos ${ }^{24}$. Para análise os resultados foram agrupados sem excesso de peso (baixo peso e peso adequado) e com excesso de peso (sobrepeso e obesidade).

A aferição da pressão arterial foi realizada pelo método oscilométrico, com uso do aparelho automático G-TECH, modelo MA 100, no braço esquerdo, com o entrevistado sentado confortavelmente, com os pés apoiados no chão, e braçadeira ao nível do coração. Foram feitas três medidas com intervalo de 2 minutos entre cada uma delas, registrando-se a média das mesmas. Para a classificação da HAS utilizou-se a definição da VI Diretriz Brasileira de Hipertensão Arterial, que considera como hipertensão, a elevação sustentada dos níveis pressóricos, correspondendo a $\geq 140 \mathrm{~mm} \mathrm{Hg}$ para a Pressão Arterial Sistólica e/ ou $\geq 90 \mathrm{~mm}$ Hg para a Pressão Arterial Diastólica, em indivíduos com idade mínima de 18 anos, obtida por medida casual, na ausência de medicação anti-hipertensiva ${ }^{25}$. Adicionalmente, na PNS, indivíduos que apresentaram níveis tensionais normais, mas que referiram uso de medicação anti-hipertensiva também foram considerados hipertensos.

\section{Análise dos dados}

As análises estatísticas foram desenvolvidas com auxílio do software Statistical Package for the Social Science (SPSS for Windows - versão 19.0), utilizando o módulo Complex Sample e considerando as ponderações utilizadas pelo delineamento amostral do estudo para a análise de dados provenientes de amostra complexa.

$\mathrm{Na}$ análise bivariada foi utilizado o teste do qui-quadrado e em seguida, a associação entre os fatores avaliados e a frequência de problemas com o sono foi estimada por meio de modelos de regressão logística multinominal (Odds Ratio [OR] e Intervalo de Confiança de 95\% [IC95\%]), estratificados por sexo e ajustados por idade, considerando como referência a categoria "Nenhum dia”. 


\section{Aspectos éticos}

O inquérito foi aprovado pela Comissão Nacional de Ética em Pesquisa para Seres Humanos, do Ministério da Saúde. O Termo de consentimento Livre e Esclarecido foi firmado no próprio PDA. Todos os Termos de Consentimento Livre e Esclarecido estão disponíveis no sítio eletrônico da PNS.

\section{Resultados}

No presente estudo foram avaliados 60.202 indivíduos, sendo 52,9\% do sexo feminino, 34,2\% estavam na faixa etária de 40 a 59 anos, 14,7\% relataram uso atual de tabaco, $13,7 \%$ apresentaram consumo abusivo de bebidas alcoólicas, 68,5\% não praticam atividade física, 28,9\% apresentaram comportamento sedentário, 32,3\% tinham hipertensão arterial e 53,8\%, excesso de peso corporal (Tabela 1).

Com relação aos problemas com o sono, foi observado que $71,1 \%$ dos indivíduos relataram não ter essa condição em nenhum dos dias nas últimas duas semanas, contudo, 14,0\% relatou ter problemas com o sono em pelo menos metade dos dias e 14,9\% tinham problemas com o sono em mais da metade dos dias (Tabela 1).

$\mathrm{Na}$ análise bivariada foi possível verificar que a ocorrência de problemas com o sono em "Mais da metade dos dias" foi mais frequente entre as mulheres $(18,2$ vs $11,2 \%, \mathrm{p}<0,01)$, com o aumento da idade (18-24 anos: 10,0\%, 25-39 anos: $12,0 \%, 40-59$ anos: $17,6 \%, 60$ anos ou mais: $19,3 \%, \mathrm{p}<0,01)$, entre indivíduos com o hábito de fumar $(19,4$ vs $14,1 \%, \mathrm{p}>0,01)$, com inatividade física $(16,1$ vs $12,4 \%, \mathrm{p}<0,01)$, com o comportamento sedentário $(17,1$ vs $14,0 \%, \mathrm{p}<0,01)$, com hipertensão arterial $(19,4$ vs $12,7 \%, \mathrm{p}<0,01)$ e com excesso de peso corporal (15,8 vs 13,8\%, $\mathrm{p}<0,01)$. Contudo, não foi associada ao consumo de bebidas alcoólicas $(\mathrm{p}=0,46)$ (Tabela 1$)$.

Por meio dos modelos de regressão logística multinomial verificou-se que, para homens e mulheres, respectivamente, maiores chances de apresentar problemas com o sono em Mais da metade dos dias foram observadas entre os tabagistas ( $\mathrm{OR}=1,55$ e 1,69), com consumo abusivo de bebidas alcoólicas $(\mathrm{OR}=1,33$ e 1,45), fisicamente inativos $(\mathrm{OR}=1,20$ e 1,13$)$, com comportamentos sedentários $(\mathrm{OR}=1,17$ e 1,22$)$ e hipertensão arterial $(\mathrm{OR}=1,33$ e 1,50). E, também, entre as mulheres com excesso de peso corporal $(\mathrm{OR}=1,17)$ (Tabela 2).

\section{Discussão}

Aproximadamente $15 \%$ da população brasileira apresentou problemas com o sono em mais da metade dos dias nas duas semanas anteriores a realização da Pesquisa Nacional de Saúde. Ser do sexo feminino, o aumento da idade, apresentar comportamentos de risco relacionados ao estilo de vida e ter hipertensão arterial e excesso de peso corporal aumentou as chances de ter problemas com o sono.

Diversos estudos têm destacado a importância da duração e da qualidade do sono para a promoção da saúde e bem-estar, assim como os efeitos deletérios à saúde acarretados por problemas com o sono. Nesse contexto, as principais consequências associadas aos problemas com o sono tem sido a redução da qualidade de vida, alto absenteísmo, aumento do risco de acidentes, elevados custos associados aos cuidados com a saúde, prejuízo cognitivo (principalmente em crianças e adolescentes), alterações emocionais e o desenvolvimento de doenças crônicas como obesidade, diabetes e hipertensão arterial ${ }^{26-31}$.

Linton et al. ${ }^{6}$, em estudo de revisão sistemática, apontam que os distúrbios do sono têm sido o transtorno primário em saúde para os trabalhadores em países industrializados. Segundo esses autores, fatores psicossociais do trabalho tais como elevada demanda de trabalho, estresse no trabalho, assédio moral e desequilíbrio na díade esforço-recompensa foram positivamente relacionados ao desenvolvimento de problemas com o sono; por outro lado, apoio social, controle e justiça organizacional no trabalho são fatores psicossociais relacionados a redução desses problemas $^{6}$.

A frequência de problemas com o sono em "Mais da metade dos dias" foi maior em mulheres, semelhante ao observado por Krishnan e Collop ${ }^{32}$, que verificaram, em estudo de revisão, que mulheres possuíam pior qualidade de sono quando comparado aos homens, podendo ser justificado por variações hormonais e fisiológicas (puberdade, menstruação, gravidez e menopausa). Além disso, as alterações na qualidade do sono podem estar mais presentes na população feminina devido à sobrecarga de trabalho desempenhados por elas (mãe/dona de casa e profissional), fazendo assim com que os momentos de descansos sejam voltados para realização de tarefas ${ }^{9}$.

Ohayon e Lemoine ${ }^{33}$ verificaram que as mulheres francesas, mesmo repousando mais cedo e acordando mais tarde, levam mais tempo para adormecer em comparação aos homens de idade 
Tabela 1. Distribuição da frequência de problemas com o sono e sua associação com as variáveis de interesse. Pesquisa Nacional de Saúde, Brasil, 2013.

\begin{tabular}{|c|c|c|c|c|c|}
\hline \multirow{3}{*}{ Característica da população } & \multirow{3}{*}{$\begin{array}{l}\text { Total } \\
\text { N (\%) }\end{array}$} & \multicolumn{3}{|c|}{ Frequência de problemas com o sono } & \multirow{3}{*}{$\begin{array}{l}\text { Valor } \\
\text { de p }\end{array}$} \\
\hline & & Nenhum dia & $\begin{array}{c}\text { Menos da } \\
\text { metade dos dias }\end{array}$ & $\begin{array}{c}\text { Mais da metade } \\
\text { dos dias }\end{array}$ & \\
\hline & & \multicolumn{3}{|c|}{$\%($ IC95\%) } & \\
\hline Total & 60.202 & $71,1(70,5 ; 71,7)$ & $14,0(13,5 ; 14,5)$ & $14,9(14,4 ; 15,4)$ & \\
\hline Sexo & & & & & $<0,01$ \\
\hline Masculino & 47,1 & $76,5(75,5 ; 77,3)$ & $12,3(11,6 ; 13,0)$ & $11,2(10,6 ; 11,9)$ & \\
\hline Feminino & 52,9 & $66,4(65,5 ; 67,2)$ & $15,4(14,8 ; 16,1)$ & $18,2(17,5 ; 18,9)$ & \\
\hline Idade $(\mathrm{em} \text { anos })^{\star}$ & & & & & $<0,01$ \\
\hline $18-24$ & 15,9 & $78,7(77,1 ; 80,3)$ & $11,2(10,1 ; 12,5)$ & $10,0(8,9 ; 11,2)$ & \\
\hline $25-39$ & 31,8 & $74,9(73,9 ; 75,9)$ & $13,1(12,3 ; 13,8)$ & $12,0(11,3 ; 12,7)$ & \\
\hline $40-59$ & 34,2 & $66,9(65,7 ; 68,0)$ & $15,6(14,7 ; 16,5)$ & $17,6(16,7 ; 18,5)$ & \\
\hline 60 ou mais & 18,0 & $65,7(64,2 ; 67,2)$ & $14,9(13,8 ; 16,1)$ & $19,3(18,1 ; 20,7)$ & \\
\hline Tabagismo & & & & & $<0,01$ \\
\hline Não & 85,3 & $71,9(71,3 ; 72,6)$ & $13,9(13,4 ; 14,5)$ & $14,1(13,6 ; 14,7)$ & \\
\hline Sim & 14,7 & $66,3(64,6 ; 68,1)$ & $14,2(13,0 ; 15,5)$ & $19,4(18,0 ; 21,0)$ & \\
\hline Consumo de bebida alcoólica & & & & & 0,46 \\
\hline Não & 86,3 & $72,0(70,4 ; 73,7)$ & $13,3(12,2 ; 14,6)$ & $14,6(13,4 ; 15,9)$ & \\
\hline Sim & 13,7 & $71,0(70,3 ; 71,6)$ & $14,1(13,6 ; 14,6)$ & $15,0(14,4 ; 15,5)$ & \\
\hline Inatividade física & & & & & $<0,01$ \\
\hline Não & 31,5 & $73,1(72,0 ; 74,2)$ & $14,5(13,7 ; 15,4)$ & $12,4(11,6 ; 13,2)$ & \\
\hline Sim & 68,5 & $70,2(69,4 ; 70,9)$ & $13,7(13,2 ; 14,3)$ & $16,1(15,5 ; 16,7)$ & \\
\hline Comportamento sedentário & & & & & $<0,01$ \\
\hline Não & 71,1 & $71,7(71,0 ; 72,5)$ & $14,2(13,7 ; 14,8)$ & $14,0(13,5 ; 14,6)$ & \\
\hline Sim & 28,9 & $69,6(68,5 ; 70,8)$ & $13,3(12,5 ; 14,1)$ & $17,1(16,1 ; 18,1)$ & \\
\hline Hipertensão arterial & & & & & $<0,01$ \\
\hline Não & 67,7 & $73,9(73,1 ; 74,6)$ & $13,4(12,9 ; 14,0)$ & $12,7(12,1 ; 13,3)$ & \\
\hline Sim & 32,3 & $65,5(64,3 ; 66,7)$ & $15,1(14,2 ; 16,0)$ & $19,4(18,4 ; 20,4)$ & \\
\hline Excesso de peso corporal & & & & & $<0,01$ \\
\hline Não & 46,2 & $72,5(71,6 ; 73,4)$ & $13,7(13,0 ; 14,4)$ & $13,8(13,1 ; 14,5)$ & \\
\hline Sim & 53,8 & $70,0(69,1 ; 70,9)$ & $14,2(13,6 ; 14,9)$ & $15,8(15,1 ; 16,5)$ & \\
\hline
\end{tabular}

equivalente. Além disso, a frequência do despertar noturno dessas mulheres foi maior, levando assim a pior qualidade do sono. No Brasil, em estudo realizado por Zanuto et al. ${ }^{4}$, também foi observado que as alterações no sono foram mais reportadas por mulheres, pessoas mais velhas e com obesidade. Por outro lado, pessoas com maior escolaridade e fisicamente ativas apresentaram menor ocorrência do desfecho analisado.

Em estudo realizado em Santos-SP, Moreira et al. ${ }^{9}$ verificaram que mulheres normalmente apresentavam mais queixas, como insônia, e relacionaram esse resultado com as variações hormonais do organismo feminino. Já o sexo masculino apresentou em maior frequência problemas respiratórios relacionados ao sono, como exem- plo a apneia, além disso, foram os mais queixosos quanto a sonolência diurna excessiva9.

Outra característica que afeta diretamente a qualidade do sono é a idade. No presente estudo constatou-se que com o aumento da faixa etária houve aumento na frequência de problemas com o sono em "Mais da metade dos dias". Resultados semelhantes foram observados por Ohayon e Lemoine ${ }^{33}$ ao verificarem que com o envelhecer, o horário de dormir tornou-se mais cedo e o de despertar, mais tarde, contudo, as alterações como a interrupção e qualidade do sono não foram adequadas, principalmente em indivíduos com 75 anos ou mais.

As dificuldades para dormir afetam indivíduos de todas as idades, mas há um aumento no 
Tabela 2. Fatores associados à frequência de problemas com o sono $(\mathrm{N}=60.202)$. Pesquisa Nacional de Saúde, Brasil, 2013.

\begin{tabular}{|c|c|c|c|c|}
\hline \multirow{4}{*}{ Característica da população } & \multicolumn{4}{|c|}{ Frequência de problemas com o sono } \\
\hline & \multicolumn{2}{|c|}{ Homens } & \multicolumn{2}{|c|}{ Mulheres } \\
\hline & $\begin{array}{c}\text { Menos da } \\
\text { metade dos dias }\end{array}$ & $\begin{array}{l}\text { Mais da metade } \\
\text { dos dias }\end{array}$ & $\begin{array}{c}\text { Menos da } \\
\text { metade dos dias }\end{array}$ & $\begin{array}{c}\text { Mais da metade } \\
\text { dos dias }\end{array}$ \\
\hline & \multicolumn{4}{|c|}{ Odds Ratio $($ IC95\%)* } \\
\hline \multicolumn{5}{|l|}{ Tabagismo } \\
\hline Não & 1 & 1 & 1 & 1 \\
\hline Sim & $1,08(0,92 ; 1,28)$ & $1,55(1,32 ; 1,83)$ & $1,25(1,07 ; 1,47)$ & $1,69(1,46 ; 1,95)$ \\
\hline \multicolumn{5}{|l|}{ Consumo de bebida alcoólica } \\
\hline Não & 1 & 1 & 1 & 1 \\
\hline Sim & $1,04(0,90 ; 1,21)$ & $1,33(1,14 ; 1,55)$ & $1,32(1,07 ; 1,63)$ & $1,45(1,20 ; 1,74)$ \\
\hline \multicolumn{5}{|l|}{ Inatividade física } \\
\hline Não & 1 & 1 & 1 & 1 \\
\hline $\operatorname{Sim}$ & $0,93(0,81 ; 1,06)$ & $1,20(1,03 ; 1,39)$ & $0,87(0,77 ; 0,97)$ & $1,13(1,00 ; 1,27)$ \\
\hline \multicolumn{5}{|l|}{ Comportamento sedentário } \\
\hline Não & 1 & 1 & 1 & 1 \\
\hline Sim & $0,92(0,79 ; 1,06)$ & $1,17(1,01 ; 1,35)$ & $0,95(0,85 ; 1,06)$ & $1,22(1,10 ; 1,35)$ \\
\hline \multicolumn{5}{|l|}{ Hipertensão arterial } \\
\hline Não & 1 & 1 & 1 & 1 \\
\hline $\operatorname{Sim}$ & $1,15(1,00 ; 1,33)$ & $1,33(1,14 ; 1,55)$ & $1,10(0,97 ; 1,25)$ & $1,50(1,34 ; 1,69)$ \\
\hline \multicolumn{5}{|l|}{ Excesso de peso corporal } \\
\hline Não & 1 & 1 & 1 & 1 \\
\hline Sim & $0,99(0,87 ; 1,13)$ & $1,04(0,91 ; 1,18)$ & $1,08(0,97 ; 1,20)$ & $1,17(1,05 ; 1,29)$ \\
\hline
\end{tabular}

número de afetados diretamente proporcional ao aumento da idade das pessoas ${ }^{34}$. Dores, uso de medicamentos e diferentes condições clínicas são exemplos de fatores que podem afetar a quantidade e a qualidade do sono entre idosos, que são mais propensos a essas condições ${ }^{35}$.

No presente estudo, foi possível observar que os quatro comportamentos de risco relacionados ao estilo de vida permaneceram associados a ocorrência de problemas com o sono em "Mais da metade dos dias", tanto nos homens quanto para as mulheres.

O estilo de vida é considerado como um ponto primordial para a qualidade do sono. Moreira et al. ${ }^{9}$ observaram que pessoas que eram fisicamente ativas possuíam melhor qualidade do sono quando comparados os indivíduos sedentários. Os autores destacam ainda que a prática de atividade física é considerada pela American Sleep Disorders Association como uma importante intervenção não farmacológica, que causa alterações positivas no perfil fisiológico, bioquímico e psicológico e está relacionada com a qualidade de vida e com o retardo de aspectos do envelhecimento.
No presente estudo, foi observado que a maioria das pessoas que relatam alterações no sono em mais da metade dos dias não praticavam atividade física regularmente. Um estudo realizado por Seixas et al. ${ }^{11}$ mostrou que a duração do sono e a prática de atividade física estão associadas à redução do risco de ter acidente vascular cerebral. Pessoas que tem um sono de 7-8 horas e praticam de 30 a 60 minutos de atividade física moderada (caminhadas, ciclismo e trabalhos leves) diariamente, apresentaram menor risco de terem um derrame ${ }^{11}$.

Outro comportamento relacionado ao estilo de vida que foi positivamente associado à maior frequência de problemas com o sono foi o tabagismo. Essa associação pode ser explicada pelo efeito da nicotina, que estimula a liberação de neurotransmissores (dopamina e serotonina), resultando em sono perturbado. A nicotina afeta ainda a função pulmonar e promove inflamação das vias aéreas, o que eleva à predisposição para roncos e apneia, sendo que esse efeito pode persistir por várias horas após seu uso ${ }^{36}$.

De acordo com Leproult e Cauter ${ }^{37}$ a propensão ao sono e a arquitetura do sono são con- 
troladas pela interação de dois mecanismos de controle do tempo no sistema nervoso central, o ritmo circadiano (ou seja, efeitos intrínsecos do tempo biológico) e homeostase do sono-vigília. Os principais mecanismos pelos quais os efeitos moduladores do ritmo circadiano e da homeostase do sono-vigília são exercidos nos sistemas fisiológicos periféricos incluem a modulação dos fatores ativadores e inibidores hipotalâmicos que controlam a liberação de hormônios hipofisários e a modulação da atividade nervosa simpática e parassimpática.

Dessa forma, a qualidade do sono é um componente importante de vulnerabilidade da saúde humana, pois a arquitetura e fisiologia do sono são processos ativos, complexos e necessários para o estabelecimento da saúde física e cogniti$\mathrm{va}^{38}$. Problemas no sono, de modo geral, estão associados com maior ocorrência de doenças metabólicas e cardiovasculares. No presente estudo, indivíduos com hipertensão arterial e excesso de peso corporal tiveram maiores chances de apresentar problemas com o sono.

Dados do Behavioural Risk Factor Surveillance System apontam que distúrbios do sono $(<5$ horas de sono por dia e tempo insuficiente de sono por dia durante a semana) está associado a desfechos de saúde cardiometabólico ${ }^{29}$. Nesse estudo, Altman et al. ${ }^{29}$ observaram que esses distúrbios, mesmo após ajuste para confundidores, permaneceram associados a aumento do índice de massa corporal, obesidade, hipertensão arterial, ataque cardíaco e acidente vascular cerebral.

No BiDirect Study, realizado em Münster, na Alemanha, Rahe et al. ${ }^{31}$ observaram que a pior qualidade do sono foi associada ao aumento da obesidade e da gordura corporal total, mesmo após ajuste para fatores sócio demográficos e de estilo de vida. No Brasil, dados do estudo ISACamp 2008/2009, apontam que tanto o menor tempo de sono ( $\leq 6$ horas) quanto o maior $(\geq 9$ horas) foram mais prevalentes entre indivíduos com doenças cardíacas, problemas vasculares, reumatismo/artrite/artrose, osteoporose ou problemas emocionais, assim como o menor tempo de sono foi mais prevalente entre indivíduos que tinha três ou mais DCNT ${ }^{30}$.

De forma semelhante, Del Brutto et al. ${ }^{39}$ ao avaliarem indivíduos da zona rural do Equador, verificaram que a má qualidade do sono foi associada à presença de indicadores de risco cardiovascular, de acordo com itens propostos pela American Heart Association para avaliação da saúde cardiovascular. Em um estudo realizado nos Estados Unidos, Patel et al. ${ }^{40}$ verificaram as- sociação entre a duração do sono e a obesidade em adultos mais velhos, sendo que aqueles que relataram sono curto ( $<5$ horas) eram mais propensos a ter comorbidades. A probabilidade de ter obesidade foi 3,7 vezes maior em homens e 2,3 vezes maior em mulheres que dormiam menos de 5 anos em relação aos que dormem de 7 a 8 horas $^{40}$.

Taheri et al. ${ }^{41}$ demonstraram que os participantes com sono curto apresentaram leptina reduzida e grelina elevada. Essas diferenças nos níveis de leptina e grelina provavelmente aumentam o apetite, podendo assim explicar o ganho de peso observado com a curta duração do sono. Tais resultados são consistentes com a hipótese de que as restrições do sono alteram os níveis circulantes de hormônios metabolicamente relevantes, como a leptina e a grelina, resultando em alterações da homeostase da glicose e regulação do apetite $\mathrm{e}^{2,14,41}$.

Embora o mecanismo desse efeito ainda não seja bem estabelecido, alterações no sono já foi associada a hormônios metabólicos alterados (leptina e grelina) ${ }^{2,42}$, balanço energético ${ }^{43,44}$ e tempo disponível para comer ${ }^{43}$. Estudos anteriores também mostraram que a curta duração do sono está associada a um maior consumo de alimentos ricos em calorias ${ }^{45,46}$.

Uma possível limitação do presente estudo trata-se da forma de avaliação dos problemas com o sono. Diferentes métodos diagnósticos podem ser utilizados na investigação dos distúrbios do sono, desde exames mais sofisticados até avaliações subjetivas por meio de questionários específicos ${ }^{47}$. Apesar de não ser um método direto de avaliação da qualidade do sono, tal como a polissonografia ${ }^{47}$, ou instrumento indireto validado como o Índice de Qualidade do Sono de Pittsburg (Pittsburgh Sleep Quality Index) ${ }^{48}$, as pesquisas epidemiológicas, em sua maioria, utilizam questionários para avaliar os hábitos de sono, pois considerando a coleta de dados em larga escala, métodos mais objetivos (por exemplo, polissonografia e actigrafia) tornariam os estudos demasiadamente caros e metodologicamente inviáveis. Dessa forma, a pergunta utilizada no presente estudo pode ser considerada uma forma simplificada de avaliação da qualidade do sono. Instrumentos simplificados, avaliando condições com base nas percepções dos indivíduos, têm sido frequentemente validados e utilizados em investigações epidemiológicas como indicador útil na triagem de populações, como por exemplo, a autopercepção do estado de saúde, utilizado na predição de morbi-mortalidade ${ }^{49-51}$. 
Por fim, conclui-se que foi elevada a proporção de brasileiros que relataram ter problemas com o sono na Pesquisa Nacional de Saúde. Atenção especial deve ser dada aos grupos mais suscetíveis ao desenvolvimento de problemas com o sono, tais como mulheres, indivíduos com mais idade, com comportamentos de risco relacionados ao estilo de vida, hipertensão arterial e excesso de peso corporal.

\section{Colaboradores}

Todos os autores certificam a participação na concepção do manuscrito para tornar pública a nossa responsabilidade pelo seu conteúdo. 


\section{Referências}

1. Roeths T, Roth T. Sleep-wake state and memory function. Sleep 2000; 23(Supl. 3):S64-S68.

2. Medic G, Wille M, Hemels ME. Short- and long-term health consequences of sleep disruption. Nat Sci Sleep 2017; 9:151-161.

3. Harvey AL, Stinson K, Whitaker KL, Moskovitz D, Virk $\mathrm{H}$. The subjective meaning of sleep quality: A comparison of individuals with and without insomnia. Sleep 2008; 31(3):383-393.

4. Zanuto EAC, Lima MCS, Araújo RG, Silva EP, Anzolin CC, Araujo MYC, Codogno JS, Christofaro DGD, Fernandes RA. Distúrbios do sono em adultos de uma cidade do Estado de São Paulo. Rev Bras Epidemiol 2015; 18(1):42-53.

5. Müller MR, Guimarães SS. Impacto dos transtornos do sono sobre o funcionamento diário e a qualidade de vida. Estudos Psicol 2007; 24(4):519-528.

6. Linton SJ, Kecklund G, Franklin KA, Leissner LC, Sivertsen B, Lindberg E, Svensson AC, Hansson SO, Sundin Ö, Hetta J, Björkelund C, Hall C. The effect of the work environment on future sleep disturbances: a systematic review. Sleep Med Rev 2015; 23:10-19.

7. Barclay NL, Eley TC, Buysse DJ, Rijsdijk FV, Gregory AM. Genetic and environmental influences on different components of the Pittsburgh Sleep Quality Index and their overlap. Sleep 2010; 33(5):659-668.

8. Lallukka T, Sares-Jäske L, Kronholm E, Sääksjärvi K, Lundqvist A, Partonen T, Rahkonen O, Knekt P. Sociodemographic and socioeconomic differences in sleep duration and insomnia-related symptoms in Finnish adults. BMC Public Health 2012; 12:565.

9. Moreira LP, Ferreira GS, Virmondes L, Silva AG, Rocco DDFM. Comparação da qualidade do sono entre homens e mulheres ativos fisicamente. Rev Eletrônica Saúde Ciên 2013; 3(2):38- 49.

10. St-Onge MP, Grandner MA, Brown D, Conroy MB, Jean-Louis G, Coons M, Bhatt DL, American Heart Association Obesity, Behavior Change, Diabetes, and Nutrition Committees of the Council on Lifestyle and Cardiometabolic Health, Council on Cardiovascular Disease in the Young; Council on Clinical Cardiology, and Stroke Council. Sleep Duration and Quality: Impact on Lifestyle Behaviors and Cardiometabolic Health: A Scientific Statement from the American Heart Association. Circulation 2016; 134(18):e367-e386.

11. Seixas AA, Vallon J, Barnes-Grant A, Butler M, Langford AT, Grandner MA, Schneeberger AR, Huthchinson J, Zizi F, Jean-Louis G. Mediating effects of body mass index, physical activity, and emotional distress on the relationship between short sleep and cardiovascular disease. Medicine (Baltimore) 2018; 97(37):e11939.

12. Markwald RR, Melanson EL, Smith MR, Higgins J, Perreault L, Eckel RH, Wright KP Jr. Impact of insufficient sleep on total daily energy expenditure, food intake, and weight gain. Proc Natl Acad Sci USA 2013; 110(14):5695-5700.

13. Fatima Y, Doi SAR, Mamun AA. Longitudinal impact of sleep on overweight and obesity in children and adolescents: a systematic review and bias-adjusted meta-analysis. Obesity Reviews 2015; 16(2):137-149.
14. Shankar A, Syamala S, Kalidindi S. Insufficient rest or sleep and its relation to cardiovascular disease, diabetes and obesity in a national, multiethnic sample. PLoS One 2010; 5:e14189.

15. Okubo N, Matsuzaka M, Takahashi I, Sawada K, Sato S, Akimoto N, Umeda T, Nakaji S, Hirosaki University Graduate School of Medicine. Relationship between self-reported sleep quality and metabolic syndrome in general population. BMC Public Health 2014; 14:562.

16. Cappuccio FPF, D’Elia L, Strazzullo P, Miller MA. Quantity and Quality of Sleep and Incidence of Type 2 Diabetes A systematic review and meta-analysis. Diabetes Care 2010; 33(2):414-420.

17. Bittencourt LR, Santos-Silva R, Taddei JA, Andersen ML, Mello MT, Tufik S. Sleep complaints in the adult Brazilian population: a national survey based on screening questions. J Clin Sleep Med 2009; 5(5):459-463.

18. Hirotsu C, Bittencourt L, Garbuio S, Andersen ML, Tufik S. Sleep complaints in the Brazilian population: Impact of socioeconomic factors. Sleep Science 2014; 7(3):135-142.

19. Instituto Brasileiro de Geografia e Estatística (IBGE). Pesquisa Nacional de Saúde 2013: percepção do estado de saúde, estilos de vida e doenças crônicas. Rio de Janeiro: IBGE; 2014.

20. Szwarcwald CL, Malta DC, Pereira CA, Vieira MLFP, Conde WL, Souza Júnior PRB, Damacena GN, Azevedo LO, Silva GA, Theme Filha MM, Lopes CS, Romero DE, Almeida WS, Monteiro CA. Pesquisa Nacional de Saúde no Brasil: concepção e metodologia de aplicação. Cien Saude Colet 2014; 19(2):333-342.

21. Damacena GN, Szwarcwald CL, Malta DC, Souza Júnior PRB, Vieira MLFP, Pereira CA, Morais Neto OL, Silva Júnior JB. O processo de desenvolvimento da Pesquisa Nacional de Saúde no Brasil, 2013. Epidemiol Serviços Saúde 2015; 24(2):197-206.

22. Onis M, Onyango AW, Borghi E, Siyam A, Nishida C, Siekmann J. Development of a WHO growth reference for school-aged children and adolescents. Bull World Health Organ 2007; 85(9):660-667.

23. World Health Organization (WHO). Obesity: preventing and managing the global epidemic. Geneva: WHO; 2000.

24. The Nutrition Screening Initiative. Incorporating $\mathrm{Nu}$ trition Screening and Interventions into Medical Practice: A Monograph for Physicians. Whashington: The Nutrition Screening Initiative; 1994.

25. Sociedade Brasileira de Cardiologia (SBC). 7a Diretriz Brasileira de Hipertensão Arterial. Arq Bras Cardiol 2016; 107:1-103.

26. Marshall NS, Glozier N, Grunstein RR. Is sleep duration related to obesity? A critical review of the epidemiological evidence. Sleep Med Rev 2008; 12(4):289298.

27. Ohayon MM, Guilleminault C, Chokroverty S. Sleep epidemiology 30 years later: where are we? Sleep Med 2010; 11(10):961-962.

28. Park S, Cho MJ, Chang SM, Bae JN, Jeon HJ, Cho SJ, Kim BS, Chung IW, Ahn JH, Lee HW, Hong JP. Relationships of sleep duration with sociodemographic and health-related factors, psychiatric disorders and sleep disturbances in a community sample of Korean adults. J Sleep Res 2010; 19(4):567-577. 
29. Altman NG, Izci-Balserak B, Schopfer E, Jackson N, Rattanaumpawan P, Gehrman PR, Patel NP, Grandner MA. Sleep duration versus sleep insufficiency as predictors of cardiometabolic health outcomes. Sleep Med 2012; 13(10):1261-1270.

30. Lima MG, Bergamo FPM, Barros MBA. Sleep duration pattern and chronic diseases in Brazilian adults (ISACAMP, 2008/09). Sleep Med 2012; 13(2):139-144.

31. Rahe C, Czira ME, Teismann H, Berger K. Associations between poor sleep quality and different measures of obesity. Sleep Med 2015; 16(10):1225-1228.

32. Krishnan V, Collop NA. Gender differences in sleep disorders. Curr Opin Pulm Med 2006; 12(6):383-389.

33. Ohayon MM, Lemoine P. Sleep and insomnia markers in the general population. Encephale 2004; 30(2):135140.

34. Oliveira BHD, Yassuda MS, Cupertino APFB, Neri AL. Relações entre padrão do sono, saúde percebida e variáveis socioeconômicas em uma amostra de idosos residentes na comunidade: Estudo PENSA. Cien Saude Colet 2010; 15(3):851-860.

35. McCrae CS, Wilson NM, Lichstein KL, Durrence HH, Taylor DJ, Bush AJ, Riedel BW. 'Young old' and 'old old' poor sleepers with and without insomnia complaints. J Psychosom Res 2003; 54(1):11-19.

36. Wetter DW, Young TB. The relation between cigarette smoking and sleep disturbance. Prev Med 1994; 23(3):328-334.

37. Leproult R, Van Cauter E. Role of sleep and sleep loss in hormonal release and metabolism. Endocr Dev 2010; 17:11-21.

38. Chokroverty S. Overview of sleep \& sleep disorders. Indian J Med Res 2010; 131:126-140.

39. Del Brutto OH, Mera RM, Zambrano M, Del Brutto VJ, Castillo PR. Association between sleep quality and cardiovascular health: A door-to-door survey in rural Ecuador. Environ Health Prev Med 2014; 19(3):234237.

40. Patel SR, Blackwell T, Redline S, Ancoli-Israel S, Cauley JA, Hillier TA, Lewis CE, Orwoll ES, Stefanick ML, Taylor BC, Yaffe K, Stone KL; Osteoporotic Fractures in Men Research Group; Study of Osteoporotic Fractures Research Group. The association between sleep duration and obesity in older adults. Int J Obes (Lond) 2008; 32(12):1825-1834.

41. Taheri S, Lin L, Austin D, Young T, Mignot E. Short sleep duration is associated with reduced leptin, elevated ghrelin, and increased body mass index. PLoS Med 2004; 1(3):e62.

42. Meng L, Zheng Y, Hui R. The relationship of sleep duration and insomnia to risk of hypertension incidence: a meta-analysis of prospective cohort studies. Hypertens Res 2013; 36(11):985-995.
43. St-Onge MP, Roberts AL, Chen J, Kelleman M, O'Keeffe M, RoyChoudhury A, Jones PJ. Short sleep duration increases energy intakes but does not change energy expenditure in normal-weight individuals. Am J Clin Nutr 2011; 94(2):410-416.

44. Caron AM, Stephenson R. Energy expenditure is affected by rate of accumulation of sleep deficit in rats. Sleep 2010; 33(9):1226-1235.

45. Nedeltcheva AV, Kilkus JM, Imperial J, Kasza K, Schoeller DA, Penev PD. Sleep curtailment is accompanied by increased intake of calories from snacks. Am J Clin Nutr 2009; 89(1):126-133.

46. Grandner MA, Kripke DF, Naidoo N, Langer RD. Relationships among dietary nutrients and subjective sleep, objective sleep, and napping in women. Sleep Med 2010; 11(2):180-184.

47. Togeiro SM, Smith AK. Diagnostics methods for sleep disorders. Rev Bras Psiquiatr 2005; 27(Supl. 1):8-15.

48. Bertolazi NA, Fagondes SC, Hoff LS, Dartora EG, Miozzo IC, Barba ME, Barreto SS. Validation of the Brazilian Portuguese version of the Pittsburgh Sleep Quality Index. Sleep Med 2011; 12(1):70-75.

49. Barreto SM, Figueiredo RCD. Doença crônica, Auto Avaliação de Saúde e comportamento de risco: diferença de gênero. Rev Saúde Pública 2009; 43(Supl. 2):38-47.

50. Kondo N, Sembajwe G, Kawachi I, Dam RM, Subramanian SV, Yamagata Z. Income inequality, mortality, and self-rated health: meta-analysis of multilevel studies. BMJ 2009; 339: b4471.

51. Guimarães JMN, Chor D, Werneck GL, Carvalho MS, Coeli CM, Lopes CS, Faerstein E. Association between self-rated health and mortality: 10 years follow-up to the Pro-saúde cohort study. BMC Public Health 2012; 12:676.

Artigo apresentado em 19/03/2020

Aprovado em 12/04/2020

Versão final apresentada em 14/04/2020

Editores chefes: Romeu Gomes, Antônio Augusto Moura da Silva 